\title{
Propiedades Psicométricas del Cuestionario de Comportamientos Interpersonales en el Deporte en deportistas universitarios de Lima
}

\author{
Sebastián Cáceres* \\ Pontificia Universidad Católica del Perú, Lima, Perú \\ https://orcid.org/0000-0002-6894-8696 \\ Rafael Gargurevich \\ Pontificia Universidad Católica del Perú, Lima, Perú \\ https://orcid.org/0000-0001-6346-4134 \\ Lennia Matos \\ Pontificia Universidad Católica del Perú, Lima, Perú \\ https://orcid.org/0000-0003-2271-4816 \\ Allison De La Haza-Gonzales \\ Pontificia Universidad Católica del Perú, Lima, Perú \\ https://orcid.org/0000-0002-5789-6042
}

\section{Resumen}

Introducción: Este cuestionario evalúa la percepción de los comportamientos interpersonales de apoyo u obstaculización de las necesidades psicológicas básicas de los deportistas (autonomía, competencia, relación) por parte de sus entrenadores(as), según la Teoría de la Autodeterminación. Objetivo: Determinar las propiedades psicométricas del Cuestionario de Comportamientos Interpersonales en el Deporte (IBQD) adaptado al Perú. Método: Participaron en el estudio 228 deportistas universitarios $\left(\mathrm{M}_{\text {edad }}=21.24 ; D E=2.39\right)$ de ambos sexos. Resultados: los análisis factoriales confirmatorios mostraron que un modelo de seis factores obtuvo buenos índices de ajuste y una adecuada confiabilidad. Los análisis correlacionales con las variables: satisfacción con la vida, afecto positivo y negativo, satisfacción y frustración de necesidades psicológicas básicas, y burnout mostraron evidencias de validez convergente y discriminante satisfactorias. Discusión: el IBQD puede ser utilizado en el contexto deportivo universitario peruano para evaluar la percepción de los comportamientos interpersonales que muestran sus entrenadores(as).

Palabras clave: Comportamiento interpersonal; Apoyo y obstaculización de necesidades; Necesidades psicológicas básicas; Propiedades psicométricas; IBQD.

\section{Psychometric Properties of the Interpersonal Behaviors Questionnaire in Sport in university athletes from Lima}

\section{Abstract}

The aim of this research was to investigate the psychometric properties of the Interpersonal Behaviors Questionnaire in Sport adapted to Peru. Based on Self-Determination Theory, this questionnaire assess the perception of interpersonal behaviors that support or thwart the basic psychological needs of athletes (autonomy, competence, relatedness) by their coaches. 
228 university athletes $\left(M_{\text {age }}=21.24 ; S D=2.39\right)$ of both sexes participated in the study. The results of the confirmatory factor analyzes showed that a six-factor model obtained good fit index and adequate reliability. Correlational analyzes with variables: life satisfaction, positive and negative affect, satisfaction and frustration of basic psychological needs, and burnout showed satisfactory evidence of convergent and discriminant validity. Thus, it was concluded that the IBQD could be used in the university sports Peruvian context to assess the perception of interpersonal behaviors displayed by its trainers.

Keywords: Interpersonal behavior; Supportive and thwarting needs; Basic psychological needs; Psychometric properties; IBQD.

\title{
Propriedades Psicométricas do Questionário de Comportamentos Interpessoais no Esporte em atletas universitários de Lima
}

\begin{abstract}
Resumo
Foram investigadas as propriedades psicométricas do Questionário de Comportamentos Interpessoais no Esporte adaptado ao Peru. Com base na Teoria da Autodeterminação, este questionário avalia a percepção de comportamentos interpessoais que apoiam ou obstaculizam as necessidades psicológicas básicas dos atletas (autonomia, competência, relacionamento) por seus treinadores. Participaram do estudo 228 atletas universitários $\left(M_{\text {idade }}=21,24 ; D P=2,39\right)$ de ambos os sexos. Os resultados das análises fatoriais confirmatórias mostraram que um modelo de seis fatores obteve bons índices de ajuste e uma confiabilidade adequada. Análises correlacionais com variáveis: satisfação com a vida, afeto positivo e negativo, satisfação e frustração das necessidades psicológicas básicas e burnout mostraram evidências satisfatórias de validade convergente e discriminante. Assim, concluiu-se que o IBQD pode ser utilizado no contexto esportivo universitário peruano para avaliar a percepção de comportamentos interpessoais demonstrados por seus treinadores.

Palavras-chave: Comportamento interpessoal; Apoio e obstaculização de necessidades; Necessidades psicológicas básicas; Propriedades psicométricas; IBQD.
\end{abstract}

Citar como:

Cáceres, S., Gargurevich, R., Matos, L., \& De La Haza-Gonzales, A. (2021). Propiedades Psicométricas del Cuestionario de Comportamientos Interpersonales en el Deporte en deportistas universitarios de Lima. Revista Digital de Investigación en Docencia Universitaria, 15(1), e1236. https://doi.org/10.19083/ridu.2021.1236

\section{Introducción}

El deporte y la vida universitaria se encuentran vinculados desde hace mucho ya que fue en 1923 que se llevaron a cabo los primeros Juegos mundiales de estudiantes universitarios en París (Tomlinson, 2010). En 1949 el vínculo universidad-deporte se vuelve más formal dada la creación de la Federación Internacional del Deporte Universitario (FISU, por sus siglas en francés) con tan solo ocho países afiliados (FISU, 2018). Sin embargo, para el 2019, ya contaba con 175 países afiliados en el mundo, siendo uno de ellos el Perú, a través de la Federación Deportiva Universitaria del Perú (FEDUP), desde 1987 (FISU, 2018; FEDUP, 2019). Estas organizaciones contribuyen en la formación integral de los estudiantes universitarios complementando el desarrollo académico con el deporte, y buscan masificar e interiorizar en la sociedad la importancia del deporte y la actividad física en la educación superior (FEDUP, 2019; Haines, 2001; Lower, Turner \& Petersen, 2013).

La importancia de la práctica deportiva dentro de la universidad radica en los beneficios que puede obtener un estudiante universitario no solo a nivel físico, sino también psicológico. Estos comprenden, por ejemplo, la mejora de la autoconfianza y autoestima, aumento del rendimiento académico, mejora de los procesos cognitivos 
relacionados al aprendizaje, mejora de las estrategias de afrontamiento frente al estrés académico, disminución de las emociones negativas (e.g. ansiedad, ira, tensión, etc.), incremento de la vitalidad, mejora las relaciones interpersonales y de la motivación (Forrester, 2015; Haines, 2001; Jang, Kim \& Reeve, 2016; Weinberg \& Gould, 2011). Estos aspectos (entre otros) han contribuido a que, en el Perú, las universidades tomen conciencia de la importancia de la actividad deportiva en el contexto universitario, dado que FEDUP cuenta, en la actualidad, con cerca de 40 universidades de todo el país participando de sus diversos campeonatos y ligas (FEDUP, 2019).

En el campo de las investigaciones en deporte, éstas se han interesado, a través de los años, en las causas psicológicas asociadas al éxito o fracaso de los deportistas, dando especial interés a la relación entre entrenadores y atletas (Bartholomew et al., 2011; Hancox et al., 2017). Ambos conviven a diario, pero son los entrenadores quienes guían al deportista en su práctica deportiva, y pueden influir en la motivación de sus dirigidos (Ntoumanis et al., 2018).

Dado que las personas obtienen información de otras personas de su entorno a través de la interacción social, resulta importante comprender el efecto que tienen los entrenadores sobre la motivación de los deportistas ya que esto afecta su desempeño en el deporte (Behzadnia et al., 2018; Deci \& Ryan, 1985; Reinboth et al., 2004).

Una de las teorías psicológicas que ha estudiado cómo los entrenadores influyen en la motivación de los deportistas es la Teoría de la Autodeterminación (Self-Determination Theory) o TAD (Ryan \& Deci, 2017). La TAD es una teoría de la motivación humana que plantea cómo los entrenadores influyen en la calidad de la motivación de sus dirigidos para practicar un deporte, disfrutarlo e involucrarse en niveles competitivos (Hagger \& Chatzisarantis, 2007; Ryan \& Deci, 2017).

Desde la TAD, la calidad de la motivación del deportista (i.e. motivación autónoma o motivación controlada) puede ser promovida u obstaculizada por el estilo motivacional de los entrenadores (Reeve, Jang \& Jang, 2018). Así, cuando un(a) entrenador(a) muestra un estilo motivacional de apoyo a la autonomía de los deportistas, y es percibido por estos últimos como tal, esto resulta en la pro- moción de la motivación autónoma, la satisfacción de necesidades psicológicas, el compromiso con el deporte, el logro de objetivos deportivos y el bienestar del deportista (Cheon \& Reeve, 2015; Gunnell et al., 2013; Jang et al., 2016). Este estilo de apoyo a la autonomía implica tomar la perspectiva del otro, crear espacios que les permitan tener iniciativa, resolver dudas con sustento lógico o racional, y reconocer y aceptar las expresiones de afecto negativo como naturales y comprensibles (Haerens et al., 2015; Reeve, 2009).

Por otro lado, cuando el(la) entrenador(a) presenta un estilo motivacional de control y es percibido por el deportista como tal, esto resulta en una motivación controlada del deportista, la frustración de las necesidades psicológicas, la deserción de la práctica deportiva y hasta en conductas antisociales (Haerens et al., 2015; Hein et al., 2015). El estilo controlador del entrenador incluye la exigencia a los deportistas de pensar, sentir y comportarse según lo dice el(la) entrenador(a) (Reeve, 2016), y no permite la opción a opinar, no se brinda explicaciones, se intimida y se intenta reprimir las expresiones de afecto negativo de los deportistas (Bartholomew et al., 2010; Reeve, 2009). En el Perú, existe ya alguna evidencia de la relación entre la percepción de estos estilos sobre la motivación de los deportistas (Garreaud, 2019; Rodríguez, 2017).

Sin embargo, la relación entre entrenadores(as) y deportistas es aún más compleja y va más allá del estudio de los estilos ya investigados en el Perú (i.e. autonomía y control), por lo cual, investigaciones recientes (Rocchi et al., 2017b; Rodrigues et al., 2019) se han centrado no solo en el clima motivacional sino en el tipo de comportamiento interpersonal que el(la) entrenador(a) muestra con el(la) deportista con respecto a cada una de las necesidades psicológicas planteadas por la TAD (Ryan \& Deci, 2017).

La TAD plantea que todas las personas poseen tres necesidades psicológicas básicas (NPB), que son autonomía, competencia y relación (Deci et al., 2006; Ryan \& Deci, 2000; Ryan et al., 2008). La necesidad de autonomía implica la libertad o voluntad de cada persona para tomar decisiones y la percepción o atribución de que la conducta responde a intereses y valores propios (Deci, La Guardia, Moller, Scheiner \& Ryan, 2006; Ryan \& 
Deci, 2000; Ryan, Patrick, Deci \& Williams, 2008). La necesidad de competencia implica sentirse capaz de poder realizar acciones progresivamente más complejas y superarlas con éxito (Deci \& Ryan, 1985; Ryan \& Deci, 2000). La necesidad de relación hace referencia al establecimiento de un vínculo emocional positivo o un lazo estrecho con personas que puedan brindar un ambiente cálido y de apoyo (Carpentier \& Mageau, 2016; Vallerand, 2001). La satisfacción de estas necesidades en cada individuo no depende solamente de elementos personales, sino de contextos interpersonales que las apoyen y no las obstaculicen ya que podrían verse frustradas (Rocchi et al., 2017b).

Es en esta relación que las conductas interpersonales del entrenador cobran importancia porque podrían apoyar u obstaculizar las necesidades psicológicas básicas (Rocchi et al., 2017b; Ryan \& Deci, 2000, 2017). Así, cuando el entrenador tiene un comportamiento interpersonal que apoya a las NPB de los deportistas, éstas se satisfacen y se obtendrán resultados positivos; mientras que, cuando el comportamiento interpersonal de los entrenadores obstaculiza las NPB de los deportistas, estas necesidades se frustran y se obtienen resultados negativos (Deci \& Ryan, 1985; Rocchi et al., 2017b; Rodrigues et al., 2019; Ryan \& Deci, 2000).

Es así que Rocchi et al. (2017b) desarrollaron el Cuestionario de Comportamientos Interpersonales en el Deporte (Interpersonal Behavior Questionnaire in Sport; IBQ-S). Basado en el Cuestionario de Comportamientos Interpersonales (IBQ; Rocchi et al., 2017a), versión adaptada al deporte, cuenta con evidencias de validez y confiabilidad en deportistas universitarios canadienses, mide el grado en el que los deportistas perciben el comportamiento interpersonal de los entrenadores, sean de apoyo o de obstaculización de sus NPB alrededor de seis subescalas del comportamiento interpersonal:

1. Apoyo a la autonomía (AA): el(la) entrenador(a) brinda opciones, explica razones de las tareas, reconoce la perspectiva del otro, da oportunidades de iniciativa y promueve el compromiso con las actividades;

2. Apoyo a la competencia (AC): el(la) entrenador(a) presenta expectativas positivas, aprendizaje alentador, feedback consistente y orientado a la promoción o al cambio, reconoce la evolución, cree en la capacidad del otro para alcanzar metas y alienta a otros a mejorar sus habilidades;

3. Apoyo a la relación (AR): el(la) entrenador(a) muestra comprensión, soporte y cuidado del otro, calidez, interesándose en sus acciones;

4. Obstaculización de la autonomía (OA): el(la) entrenador(a) usa recompensas condicionadas, lenguaje intimidante, exige sin brindar una explicación razonable y ejerce un control personal excesivo;

5. Obstaculización de la competencia (OC): el(la) entrenador(a) destaca los errores, desanima a los otros de intentar desafíos, da mensajes de incompetencia y duda de las capacidades de mejorar;

6. Obstaculización de la relación (OR): el(la) entrenador(a) es distante con los otros, no conecta emocionalmente, no escucha y no está disponible cuando se le necesita.

Este cuestionario fue utilizado por primera vez en una muestra de deportistas universitarios de ambos sexos ( $n=239 ;$ hhombres $=130$, nmujeres = 109) de la ciudad de Ottawa (Medad $=20.15 ; D E$ = 3.16) y Rocchi et al. (2017b) encontraron que el modelo de seis factores tuvo un ajuste óptimo $\left(\mathrm{SBS} \chi^{2}(237)=296.23, p<.001, \mathrm{CFI}=.95\right.$, TLI $=.95$, RMSEA = .05 [.04, .06], SRMR = .05). Encontraron un rango de carga factorial entre .69 y .87, y que la consistencia interna de cada subescala es adecuada debido a que los coeficientes alfa de Cronbach estuvieron entre .84 y .90 . Además, presentó evidencias de validez convergente y discriminante al correlacionar $(p<.001)$ las subescalas AA, AC y AR positivamente con la satisfacción de las tres NPB (de .43 a .58) y negativamente con la frustración de las NPB (de -.24 a -.40). Asimismo, las subescalas OA, OC y OR correlacionaron positivamente $(p<.001)$ con la frustración de las tres NPB (de .54 a .66) y la motivación controlada (de .41 a .51), y negativamente con la satisfacción de las NPB (de -.20 a -.44).

Por su parte, Rodrigues et al. (2019) adaptaron el IBQ en el Deporte (Rocchi et al., 2017b) al idioma portugués con deportistas que realizan ejercicios físicos en gimnasios de Portugal ( $n=837$; $n$ hom- 
bres $=342, n$ mujeres $=495)$ con una edad media de 34.58 ( $D E=11.35)$. Rodrigues et al. (2019) encontraron que el ajuste del modelo de seis factores era adecuado $\left(\mathrm{SBS} \chi^{2}(237)=828.91, p<.001, \mathrm{CFI}=\right.$ $.92, \mathrm{TLI}=.94, \mathrm{RMSEA}=.05[.05, .06], \mathrm{SRMR}=.04)$. Encontraron un rango de carga factorial entre .51 y .84, y que la consistencia interna de cada subescala es adecuada debido a que los coeficientes de confiabilidad estuvieron entre 70 y .82. Asimismo, al igual que Rocchi et al. (2017b), las subescalas de comportamiento interpersonal de apoyo a las NPB (AA, AC y AR) se relacionaron positivamente con la satisfacción de las tres NPB, y negativamente con la frustración de las mismas; mientras que el comportamiento interpersonal de obstaculización de estas NPB (OA, OC y OR) se relacionaron positivamente con la frustración de las NPB, y negativamente con la satisfacción de las mismas.

Cabe mencionar que Rocchi et al. (2017a), si bien no investigaron con el IBQ versión del Deporte, estudiaron la relación del apoyo y obstaculización de las NPB con la satisfacción de las mismas y con variables implicadas en el bienestar, tales como la satisfacción con la vida, el afecto positivo y negativo, y la vitalidad subjetiva, en una muestra de estudiantes universitarios $(n=534)$. En dicho estudio se encontraron correlaciones $(p<.001)$ positivas de las subescalas de apoyo a las NPB (AA, $A C$ y AR) con la satisfacción de éstas (de .32 a .59), el afecto positivo (de .45 a .54), la satisfacción con la vida (de .40 a .45) y la vitalidad subjetiva (de .39 a .45); y negativas ( $p<.001)$ con el afecto negativo (de -.30 a -.41). Mientras que las subescalas OA, OC y OR correlacionaron positivamente $(p<.001)$ con el afecto negativo (de .41 a .45), y negativamente $(p<.001)$ con la satisfacción de las NPB (de -.34 a -.58), el afecto positivo (de -.31 a -.46), la satisfacción con la vida (de -.33 a -.40) y la vitalidad subjetiva (de -.37 a -.46).

Por ello, dado que las propiedades psicométricas del IBQ en el Deporte se presentan sólidas, queda clara la utilidad del uso del cuestionario para evaluar los estilos interpersonales de los entrenadores(as) con respecto a los deportistas. Es así que el objetivo de este estudio es realizar la adecuación lingüística del IBQ del Deporte en el Perú y estudiar las propiedades psicométricas en un contexto de deportistas pertenecientes a equipos universitarios de universidades de Lima.
De esta manera, se espera confirmar la estructura factorial de seis factores del instrumento original (Rocchi et al., 2017b), o sino plantear modelos alternos que puedan explicar mejor la estructura del instrumento. Además, para comprobar la validez convergente y discriminante de la escala, se llevarán a cabo correlaciones con variables previamente estudiadas en el IBQD (Rocchi et al., 2017b), como es el caso de la satisfacción y frustración de las necesidades psicológicas básicas, la satisfacción con la vida, el afecto positivo y negativo, y la vitalidad subjetiva (Rocchi et al., 2017a, 2017b; Rodrigues et al., 2019). Finalmente, se utilizará el coeficiente omega de McDonald (McDonald, 1999) para el cálculo de la confiabilidad del IBQD.

\section{Método}

\section{Participantes}

La muestra de este estudio instrumental (Alarcón, 2008) estuvo compuesta por un total de 228 deportistas provenientes de 22 universidades de Lima Metropolitana de 2do a 12 do ciclo $(M=6.87$; $D E=2.62)$, cuyas edades oscilaron entre los 17 y 29 años ( $M=21.24 ; D E=2.39)$. Además, tenían alrededor de 18 meses entrenando con sus entrenadores ( $M=17.79$ meses; $D E=14.70$ ), y entrenaban en promedio alrededor de cuatro veces por semana ( $M=$ 3.63; $D E=1.23$ ), y por un promedio de 2.26 horas por entrenamiento $(D E=0.59)$. La distribución de la muestra, de acuerdo a las variables sociodemográficas género y deporte que practica, se puede observar en la Tabla 1.

\section{Instrumentos}

Ficha de datos sociodemográficos. Se recolectó información acerca de la edad, género (masculino, femenino, otro), universidad, ciclo académico, disciplina deportiva que practica, tiempo (en meses) que viene entrenando con su actual entrenador(a), cantidad de días que entrena semanalmente y la duración (en horas) de los entrenamientos.

\section{Cuestionario de Comportamientos Interperso-} nales en el Deporte. Desarrollado por Rocchi et al. (2017b), consta de 24 ítems que evalúan seis fac- 
Tabla 1

Distribución de la muestra según variables sociodemográficas: género y deporte

\begin{tabular}{|c|c|c|c|}
\hline & & $\boldsymbol{N}$ & $\%$ \\
\hline \multirow[t]{2}{*}{ Género } & Femenino & 130 & 57.02 \\
\hline & Masculino & 98 & 42.98 \\
\hline \multirow[t]{14}{*}{ Deporte } & Vóley & 54 & 23.7 \\
\hline & Futsal & 33 & 14.5 \\
\hline & Fútbol & 20 & 8.8 \\
\hline & Básquet & 20 & 8.8 \\
\hline & Natación & 20 & 8.8 \\
\hline & Taekwondo & 13 & 5.7 \\
\hline & Tenis de mesa & 11 & 4.8 \\
\hline & Wushu & 11 & 4.8 \\
\hline & Rugby & 7 & 3.1 \\
\hline & Béisbol & 7 & 3.1 \\
\hline & Atletismo & 6 & 2.6 \\
\hline & Judo & 6 & 2.6 \\
\hline & Tiro & 6 & 2.6 \\
\hline & Otros & 14 & 6.1 \\
\hline
\end{tabular}

tores: (1) Apoyo a la Autonomía (e.g. "Apoya mis decisiones"), (2) Apoyo a la Competencia (e.g. "Me dice que puedo lograr cosas"), (3) Apoyo a la Relación (e.g. "Se toma el tiempo de conocerme"), (4) Obstaculización de la Autonomía (e.g. "Me impone sus opiniones"), (5) Obstaculización de la Competencia (e.g. "Me da el mensaje de que soy incompetente"), y (6) Obstaculización de la Relación (e.g. "Es distante cuando pasamos tiempo juntos"). Los participantes responden cuán de acuerdo están con cada ítem en una escala Likert del 1 (Totalmente en desacuerdo), al 7 (Totalmente de acuerdo). Como se ha mencionado en la introducción, este instrumento cuenta con buenas propiedades psicométricas, llevadas a cabo mediante el análisis factorial confirmatorio $\left(\operatorname{SBS} \chi^{2}(237)=296.23, p\right.$ $<.001, \mathrm{CFI}=.95, \mathrm{TLI}=.95, \mathrm{RMSEA}=.05[.04, .06]$, SRMR = .05), y confiabilidad utilizando el coeficiente de consistencia interna alfa de Cronbach (rango entre.84 y .90).

Antes de la aplicación de los cuestionarios se llevó a cabo la traducción de ítems, instrucciones y opciones de respuesta del cuestionario. Para llevar a cabo la traducción y aplicación del IBQD en el Perú, se contó con la aprobación de Rocchi (comunicación personal), primera autora del artículo de la adaptación del IBQ en el Deporte (Rocchi et al., 2017b).

La traducción fue llevada a cabo tomando en cuenta las recomendaciones de los Estándares para pruebas Educativas y Psicológicas (AERA, APA \& NCME, 2014) y de la Comisión Internacional de Tests (Hambleton, 1994; Hambleton \& Zenisky, 2011; Muñiz et al., 2013). Así, un grupo de expertos docentes universitarios con conocimiento del idioma inglés, con grados de maestría o superiores, y con experiencia en investigación, fue reunido para evaluar la idoneidad de la traducción del IBQ. El resultado de esta evaluación fue positivo (100\% de consistencia entre los evaluadores) y, de esta manera, se obtuvo la versión final del instrumento.

Escala de Satisfacción y Frustración de las Necesidades Psicológicas Básicas. Originalmente, la escala consta de 24 ítems y fue desarrollada en Bélgica, China, Estados Unidos y Perú, simultáneamente, para medir la satisfacción y frustración 
de las NPB (Chen et al., 2015). Esta escala fue adaptada al contexto deportivo y reducida a 12 ítems (Delreu et al., 2019), la cual fue a su vez adaptada para el Perú por Matos y Gargurevich (2020) y ha sido utilizada en esta investigación para comprobar las evidencias de validez convergente y discriminante del IBQD (Rocchi et al., 2017b). Esta versión evalúa la Satisfacción (e.g. "Sentí que mi entrenador(a) me entrenó de la manera en que yo quería"), y la Frustración (e.g. "Me sentí obligado(a) a hacer ejercicios que yo no elegiría hacer") de las NPB, y para responder se usa una escala tipo Likert que va del 1 (Totalmente falso), al 5 (Totalmente verdadero). El análisis de factorización de ejes principales (rotación Promax) dio como resultado una estructura bifactorial (Chen et al., 2015) en donde, para los factores 1 y 2 (Satisfacción y Frustración de las NPB), se obtuvieron coeficientes de omega de McDonald de .79 [.74-.82] y .81 [.78-.85], respectivamente.

Escala de Vitalidad Subjetiva. Esta escala, desarrollada por Ryan y Frederick (1997), mide la sensación de vitalidad y energía como reflejo de bienestar psicológico y físico (e.g. "Cuando entreno, me siento vivo(a) y con energía") reunidos en un solo factor de siete ítems. El sistema de respuestas es de escala Likert que va del 1 (Nada verdadero para $\mathrm{m} \hat{1}$ ) al 7 (Totalmente verdadero para $\mathrm{m} \hat{1}$ ). En esta investigación se utilizó la escala para evaluar las evidencias de validez convergente y discriminante del IBQD (Rocchi et al., 2017b). El análisis de factorización de ejes principales dio como resultado un factor que obtuvo un coeficiente omega de McDonald de .87 [.83-.89].

Cuestionario de Agotamiento del Atleta. El Athlete Burnout Questionnaire (ABQ; Raedeke \& Smith, 2001) consta de 15 ítems distribuidos en tres factores que han sido adaptados al español (Arce et al., 2010) De Francisco, Andrade Arce, I. y Raedeke, 2010) como: (1) Agotamiento Físico y Emocional (e.g. "Practicar este deporte me deja mentalmente agotado(a)"), (2) Reducida Sensación de Logro (e.g. ítem invertido, “Creo que puedo alcanzar mis metas en el deporte") y (3) Devaluación de la Práctica Deportiva (e.g. “Creo que no me interesa tanto el deporte como antes"). Esta versión en español fue reducida a 9 ítems y mantiene las tres subes- calas del burnout deportivo (De Francisco, 2015). El sistema de respuestas es de escala Likert que mide la frecuencia de ocurrencia de los ítems, que va del 1 (Casi nunca) al 5 (Casi siempre).

Para la presente investigación se utilizó la versión reducida para evaluar las evidencias de validez convergente y discriminante del IBQD (Rocchi et al., 2017b). El análisis factorial resultó en los tres factores propuestos originalmente (De Francisco, 2015). Sin embargo, dado que la correlación entre los factores encontrados resultó bastante alta y la confiabilidad de dos factores fue baja, se llevó a cabo un segundo análisis en el que se obtuvo un solo factor que presentó un coeficiente omega de McDonald de .65 [.61-.73]. Katz (2006) indica que pueden aceptarse valores de omega de al menos .65, por lo que se decidió continuar con el uso de esta escala dado que se usaría para la comprobación de las evidencias de validez convergente y discriminante del IBQD.

\section{Escala Internacional de Afecto Positivo y Afecto} Negativo. También llamada I-PANAS-SF (Thompson, 2007), fue adaptada al Perú por Gargurevich (2010) y es utilizada en este estudio para inspeccionar las evidencias de validez convergente y discriminante del IBQD (Rocchi et al., 2017b). La prueba consta de 10 ítems repartidos equitativamente en dos factores que evalúan (1) Afecto Positivo o AP (e.g. "Inspirado(a)"), y (2) Afecto Negativo o AN (e.g. "Temeroso(a)"). Las respuestas se dan en formato de escala Likert que mide el nivel de acuerdo o desacuerdo con los ítems, desde 1 ( Totalmente en desacuerdo) hasta 5 ( Totalmente de acuerdo). El análisis de factorización de ejes principales (rotación Varimax) dio como resultado dos factores (AP y AN) y se obtuvieron coeficientes omega de McDonald de .84 [.79-.86] y .74 [.68-.79] para AN y AP, respectivamente.

Escala de Satisfacción con la Vida. Esta escala, desarrollada por Diener et al. (1985),, consta de cinco ítems (e.g. "En la mayoría de cosas, mi vida está cerca de mi ideal") que evalúan el grado de satisfacción general con la vida. Estos ítems se responden mediante una escala Likert que va del 1 (Totalmente en desacuerdo) al 5 (Totalmente de acuerdo). Esta escala ha sido adaptada en el Perú (Martínez, 2004) y ha sido utilizada en esta 
investigación para la comprobación de las evidencias de la validez convergente y discriminante del IBQD (Rocchi et al., 2017b). El análisis de factorización de ejes principales dio como resultado un factor que obtuvo un coeficiente omega de McDonald de .85 [.81-.88].

\section{Procedimientos}

Los participantes fueron contactados a través de redes sociales institucionales de las universidades con equipos deportivos y también personales (de los deportistas directamente al no haber un contacto institucional activo). Además, todos los cuestionarios fueron aplicados por medios electrónicos (online) y, antes de aplicarlos, los participantes dieron su consentimiento explícito de participación voluntaria y anónima.

\section{Análisis de datos}

Los análisis factoriales confirmatorios se realizaron mediante la Versión 8.72 del programa Linear Structural Relations (LISREL; Jöreskog \& Sörbom, 2005). Antes de llevar a cabo los AFC, se inspeccionaron la asimetría y la curtosis de los ítems de la prueba y ninguno obtuvo valores superiores a |2| (Tabachnick \& Fidell, 1996; West et al., 1995), los análisis confirmatorios se realizaron teniendo en cuenta la normalidad en la distribución de las puntuaciones. Además, se consideró que el IBQ recolectó información en una escala Likert de siete opciones de respuesta, las puntuaciones fueron tratadas como continuas (Jöreskog \& Sörbom, 1996; Maydeu-Olivares, 2017; Norman, 2010). De esta manera de utilizó el método de estimación de Máxima Verosimilitud (Jöreskog \& Sörbom, 1996).

Los resultados de los AFC fueron evaluados a través de los índices de ajuste recomendados por Hu \& Bentler (1999): chi-cuadrado $\left(\chi^{2}\right)$, raíz cuadrada media del error de aproximación (RMSEA, Root Mean Square Error of Approximation), la raíz cuadrada media residual estandarizada (SRMR, Standardized Root Mean Square Residual), y el índice de ajuste comparativo (CFI, Comparative Fit Index). Así, se compararon los chi-cuadrado $\left(\chi^{2}\right)$ de los diferentes modelos, siendo el mejor modelo el de menor valor (Kline, 2016); el RMSEA y el SRMR, donde valores de RMSEA que indican buen ajuste del modelo son valores cercanos a .07 y aceptable si es menor o igual a .08, mientras que un SRMR menor a .08 indica un excelente ajuste (Marsh et al., 2004; Simms et al., 2002; Steiger, 2007). El CFI indica un ajuste óptimo cuando obtiene un valor mayor o igual a .95, y aceptable cuando está alrededor de .90 (Hu \& Bentler, 1999; Kline, 2016).

La inspección de la estructura factorial de la prueba tomó en cuenta cuatro modelos. Así, se evaluaron: un modelo de un solo factor en el que los 24 ítems del IBQD se agrupan alrededor de una sola variable latente (Modelo 1); un modelo de dos factores reuniendo todos los ítems de apoyo y de obstaculización de las NPB (Modelo 2); el modelo original (Modelo 3 ) de seis factores (AA, $A C, A R, O A, O C$ y OR) que fue planteado por Rocchi et al. (2017b); y un Modelo 4 de dos factores de segundo orden que reunían las variables latentes del modelo 3 de seis factores, es decir, un factor de apoyo reuniendo las variables AA, AC y AR, y un factor de obstaculización reuniendo las variables OA, OC y OR.

Con respecto a la confiabilidad, se utilizó la Versión 0.13.1 del programa JASP (JASP Team, 2020). Allí se calculó utilizando el coeficiente omega de McDonald (1999), el cual ofrece un cálculo de valores precisos y estables (Ventura-León \& Caycho-Rodríguez, 2017). Finalmente, para llevar a cabo los análisis descriptivos (i.e. medias y desviaciones estándar) y las correlaciones entre las variables para lograr las evidencias de validez convergente y discriminante se usó la Versión 25 del programa IBM Statistical Package for the Social Sciences (SPSS; IBM Corp., 2017).

\section{Resultados}

\section{Evidencias de validez factorial y confiabilidad del IBQD}

Los resultados de los análisis factoiales confirmatorios de los Modelos 1, 2 y 4 no mostraron buenos índices de ajuste (ver Tabla 2). De esta manera, el modelo de seis factores (i.e. AA, AC, AR, OA, OC y OR), planteado por Rocchi et al. (2017b), resultó ser el mejor modelo para el IBQD en el contexto deportivo universitario peruano ya que presenta índices de ajuste adecuados y superiores a los de los otros modelos $\left(\chi^{2}(237)=546.66, p<.001\right.$, CFI $=$ $.93, \mathrm{RMSEA}=.08[.07, .08], \mathrm{SRMR}=.05)$. 
Cáceres, S., Gargurevich, R., Matos, L. \& De La Haza-Gonzales, A.

Tabla 2

Índices de ajuste de los modelos de AFC

\begin{tabular}{lllcccc}
\multicolumn{1}{c}{ Modelos } & \multicolumn{1}{c}{$\chi^{\mathbf{2}}$} & & gl & RMSEA & SRMR & CFI \\
\hline 1 & Un factor & $1373.22^{* * *}$ & 252 & .140 & .073 & .82 \\
2 & Dos factores & $862.04^{* * *}$ & 251 & .100 & .057 & .89 \\
3 & Seis factores & $546.66^{* * *}$ & 237 & .076 & .050 & .93 \\
4 & Dos factores (segundo orden) & $661.09^{* * *}$ & 245 & .086 & .054 & .91 \\
\hline
\end{tabular}

Nota. ${ }^{* * *} p<.001$

Todas las cargas factoriales estuvieron por encima del .40 y obtuvieron comunalidades entre .21 y .79 (ver Tabla 3). Con respecto a la confiabilidad del IBQD, el coeficiente omega de McDonald para los seis factores alcanzó un rango entre .81 y .90. El rango de correlaciones ítem-test estuvo entre .51 y .82 (ver Tabla 3). Adicionalmente, se presenta también la Varianza Media Extraída (AVE; Average Variance Extracted), donde valores cercanos o superiores a .50 muestran buena validez convegente interna (Raykov, 1997).

Asimismo, también se presentan las medias y desviaciones estándar de las escalas aplicadas (ver Tabla 4).

\section{Correlaciones entre variables: intercorrelaciones y evidencias de validez convergente y discriminante}

Al revisar las intercorrelaciones entre los factores del Modelo 3 del IBQD, se encontró que para los factores AA, AC y AR las correlaciones $(p<.001)$ oscilaron entre .80 y .81 , y para los factores OA, OC y OR, fluctuaron entre .66 y .76. Mientras que, las correlaciones entre los factores de apoyo (i.e. AA, AC y AR) y los de obstaculización (i.e. OA, OC y OR) estuvieron entre - .50 y -.76 (ver Tabla 4).

Por su parte, las correlaciones que se llevaron a cabo para comprobar las evidencias de validez convergente y discriminante resultaron ser significativas (ver Tabla 4). Así, se encontró que los tres factores de apoyo (i.e. AA, AC y AR) correlacionaron de manera positiva (entre .18 y .73) con la satisfacción de las NPB, la vitalidad subjetiva, el afecto positivo y la satisfacción con la vida, y obtuvieron correlaciones negativas (de -.25 a -.56) con la frustración de las NPB, el burnout deportivo y el afecto negativo. Mientras que los tres factores de obstaculización (i.e. OA, OC y OR) correlacionaron positivamente (entre .32 y .68) con la frustración de las NPB, el burnout deportivo y el afecto negativo, y negativamente (de -.15 a -.68) con la satisfacción de las NPB, la vitalidad subjetiva, el afecto positivo y la satisfacción con la vida.

\section{Discusión}

El objetivo de esta investigación fue estudiar, por primera vez en el Perú, las propiedades psicométricas del Cuestionario de Comportamientos Interpersonales en el Deporte (IBQD; Rocchi et al., 2017b) en el contexto peruano en una muestra de deportistas universitarios de Lima Metropolitana. Los resultados de los análisis factoriales confirmatorios realizados mostraron que la estructura original de la prueba (Rocchi et al., 2017b), de seis factores correlacionados, logró los mejores índices de ajuste en comparación a otros tres modelos competidores. Así, resulta claro que el IBQD evalúa los comportamientos interpersonales de los entrenadores(as) de apoyo o de obstaculización de cada una de las necesidades psicológicas básicas propuestas en la TAD (Ryan \& Deci, 2017).

Adicionalmente, las seis subescalas correlacionaron entre sí con la dirección esperada de acuerdo con la TAD (Ryan \& Deci, 2017) y de acuerdo con los estudios previos del IBQ en el Deporte (Rocchi et al., 2017b; Rodrigues et al., 2019). En la presente investigación, el rango de intercorrelaciones entre las subescalas del IBQD resultaron ligeramente mayores que las intercorrelaciones reportadas por Rocchi et al. (2017b), que fueron entre .70 y .74 para las de apoyo, y entre .60 y .69 para las de obstaculización, y lo mismo sucedió con las intercorrelaciones reportadas por Rodrigues et al. (2019), quienes 
Tabla 3

Cargas factoriales, comunilidades (R2), varianza media extraída (VME), coeficiente Omega de McDonald ( $\omega$ ), intervalos de confianza (IC), rango items test y estadística descriptivos del modelo de seis factores del IBQD (Modelo 3)

\begin{tabular}{|c|c|c|c|c|c|c|}
\hline & AA (R2) & OA (R2) & AC (R2) & OC (R2) & $A R(R 2)$ & OR (R2) \\
\hline IBD1 & $.70^{* * *}(.48)$ & & & & & \\
\hline IBQD7 & $.83^{* * *}(.69)$ & & & & & \\
\hline IBQD13 & $.88^{* * *}(.77)$ & & & & & \\
\hline IBQD19 & $.89^{* * *}(.79)$ & & & & & \\
\hline IBQD2 & & $.62^{* * *}(.36)$ & & & & \\
\hline IBQD8 & & $.56^{* * *}(.30)$ & & & & \\
\hline IBQD14 & & $.64^{* * *}(.37)$ & & & & \\
\hline IBQD20 & & $.88^{* * *}(.76)$ & & & & \\
\hline IBQD3 & & & $.79^{* * *}(.61)$ & & & \\
\hline IBQD9 & & & $.76^{* * *}(.56)$ & & & \\
\hline IBQD15 & & & $.86^{* * *}(.76)$ & & & \\
\hline IBQD21 & & & $.80^{* * *}(.21)$ & & & \\
\hline IBQD4 & & & & $.73^{* * *}(.49)$ & & \\
\hline IBQD10 & & & & $.85^{* * *}(.69)$ & & \\
\hline IBQD16 & & & & $.80^{* * *}(.62)$ & & \\
\hline IBQD22 & & & & $.74^{* * * *}(.53)$ & & \\
\hline IBQD5 & & & & & $.81^{* * *}(.69)$ & \\
\hline IBQD11 & & & & & $.83^{* * *}(.68)$ & \\
\hline IBQD17 & & & & & $.83^{* * *}(.69)$ & \\
\hline IBQD23 & & & & & $.76^{* * *}(.54)$ & \\
\hline IBQD6 & & & & & & $.67^{* * *}(.45)$ \\
\hline IBQD12 & & & & & & $.60^{* * *}(.43)$ \\
\hline IBQD18 & & & & & & $.81^{* * *}(.66)$ \\
\hline IBQD24 & & & & & & $.84^{* * *}(.73)$ \\
\hline AVE & .69 & .47 & .65 & .61 & .65 & .54 \\
\hline Coeficiente $\omega$ [IC] & $.90[.87-.92]$ & $.81[.76-.84]$ & $.88[.85-.91]$ & $.86[.83-.89]$ & $.89[.86-.90]$ & $.81[.75-.84]$ \\
\hline Rango ítem-test & $.69-.82$ & $.57-.68$ & $.73-.76$ & $.66-.77$ & $.70-.80$ & $.51-.73$ \\
\hline Medias $(D E)$ & $5.43(1.16)$ & $3.11(1.45)$ & $5.84(1.17)$ & $2.19(1.38)$ & $5.50(1.22)$ & $2.55(1.34)$ \\
\hline
\end{tabular}

Nota. ${ }^{* *} p<.001 ; \mathrm{AA}=$ Apoyo a la autonomía, OA = Obstaculización de la autonomía, AC = Apoyo a la competencia, OC = Obstaculización de la competencia, $A R=$ Apoyo a la relación, $\mathrm{OR}=$ Obstaculización de la relación; $\mathrm{DE}$ = Desviación estándar.

Tabla 4

Media (M) y desviación estándar (DE) de las variables de estudio

\begin{tabular}{lll} 
& M & DE \\
\hline Satisfacción de las necesidades psicológicas básicas & 4.08 & 0.69 \\
Frustración de las necesidades psicológicas básicas & 2.19 & 0.87 \\
Vitalidad subjetiva & 5.72 & 0.99 \\
Burnout deportivo & 2.25 & 0.60 \\
Afecto positivo & 4.19 & 0.54 \\
Afecto negativo & 2.37 & 0.86 \\
Satisfacción con la vida & 5.15 & 1.13 \\
\hline
\end{tabular}


Cáceres, S., Gargurevich, R., Matos, L. \& De La Haza-Gonzales, A.

Tabla 5

Correlaciones bivariadas entre las variables de estudio

\begin{tabular}{|c|c|c|c|c|c|c|}
\hline & $\mathbf{A A}$ & $\mathbf{O A}$ & AC & OC & $\mathbf{A R}$ & OR \\
\hline Apoyo a la autonomía (AA) & 1 & & & & & \\
\hline Obstaculización de la autonomía (OA) & $-.64^{* * *}$ & 1 & & & & \\
\hline Apoyo a la competencia (AC) & $.81^{* * *}$ & $-.50^{* * *}$ & 1 & & & \\
\hline Obstaculización de la competencia (OC) & $-.64^{* * *}$ & $.67^{* * *}$ & $-.69^{* * *}$ & 1 & & \\
\hline Apoyo a la relación (AR) & $.80^{* * *}$ & $-.51^{* * *}$ & $.81^{* * *}$ & $-.61^{* * *}$ & 1 & \\
\hline Obstaculización de la relación (OR) & $-.70^{* * *}$ & $.66^{* * *}$ & $-.69^{* * *}$ & $.76^{* * *}$ & $-.76^{* * *}$ & 1 \\
\hline Satisfacción de las necesidades psicológicas básicas & $.69^{* * *}$ & $-.50^{* * *}$ & $.73^{* * *}$ & $-.65^{* * *}$ & $.70^{* * *}$ & $-.68^{* * *}$ \\
\hline Frustración de las necesidades psicológicas básicas & $-.56^{* * *}$ & $.57^{* * *}$ & $-.54^{* * *}$ & $.68^{* * *}$ & $-.54^{* * *}$ & $.66^{* * *}$ \\
\hline Vitalidad subjetiva & $.49^{* * *}$ & $-.36^{* * *}$ & $.50^{* * *}$ & $-.46^{* * *}$ & $.44^{* * *}$ & $-.39 * * *$ \\
\hline Burnout deportivo & $-.34^{* * *}$ & $.37^{* * *}$ & $-.35^{* * *}$ & $.34^{* * *}$ & $-.28^{* * *}$ & $.33^{* * *}$ \\
\hline Afecto positivo & $.30^{* * *}$ & $-.24^{* * *}$ & $.32^{* * *}$ & $-.33^{* * *}$ & $.27^{* * *}$ & $-.28^{* * *}$ \\
\hline Afecto negativo & $-.33^{* * *}$ & $.35^{* * *}$ & $-.30^{* * *}$ & $.41^{* * *}$ & $-.25^{* * *}$ & $.32^{* * *}$ \\
\hline Satisfacción con la vida & $.25^{* * *}$ & $-.15^{*}$ & $.19^{* *}$ & $-.21^{* *}$ & $.18^{* *}$ & $-.15^{*}$ \\
\hline
\end{tabular}

Nota. ${ }^{*} p<.05,{ }^{* *} p<.01,{ }^{* * *} p<.001$

tuvieron un rango entre .60 y .67 para las subescalas de apoyo, y entre .66 y .71 para las de obstaculización. Rocchi et al. (2017b) mencionaron que a pesar de que las correlaciones fueron altas, no lo fueron tanto como para no poder diferenciar los seis factores hipotetizados y, de esta manera, concluyeron que las subescalas presentan constructos diferentes. En la presente investigación, este fenómeno parece repetirse dado que, al comparar el modelo de seis factores con otros modelos con un número menor de factores, el modelo de seis factores correlacionados fue el de mejor ajuste.

Además, los seis factores del IBQD obtuvieron evidencias de validez convergente y discriminante satisfactorias, lo cual es también consistente con los hallazgos previos del IBQ (Rocchi et al., 2017b; Rodrigues et al., 2019). En ambos estudios previos con el IBQD (Rocchi et al., 2017b; Rodrigues et al., 2019) se reportaron las correlaciones halladas entre cada uno de los seis tipos de comportamientos interpersonales con cada una de las seis subescalas correspondientes de satisfacción y frustración de las necesidades psicológicas básicas. En la presente investigación, estas relaciones fueron inspeccionadas con las puntuaciones totales de satisfacción y frustración de las NPB, siendo los hallazgos consistentes con los estudios previos que evaluaron apoyo u obstaculización con la satisfacción o frustración de las NPB de manera global (López-Walle et al., 2012; Vansteenkiste \& Ryan, 2013).

Así también, vale la pena mencionar que se encontraron correlaciones consistentes con investigaciones previas que no han sido llevadas a cabo con el IBQ, pero en las que se ha estudiado variables utilizadas en el presente estudio, como las correlaciones positivas entre las variables que son consideradas del bright y dark side de la motivación (Haerens et al., 2015), como lo son, por un lado, la satisfacción de las NPB, la vitalidad, el afecto positivo y la satisfacción con la vida (Bartholomew et al., 2011; Haerens et al., 2015; Garreaud, 2019; López-Walle et al., 2012; Mageau et al., 2015; Reinboth et al., 2004; Rodríguez, 2017; Sevil, Abós, Sanz \& García-González, 2018); y, por otro lado, la frustración de las NPB, el afecto negativo y el burnout (Bartholomew et al., 2011; Haerens et al., 2015 Garreaud, 2019; Mageau et al., 2015; Rodríguez, 2017; Sevil et al., 2018).

Además, la confiabilidad de cada subescala del IBQD fue bastante aceptable, lo que es consistente con los estudios previos (Rocchi et al., 2017b; Rodrigues et al., 2019). Además, en esta investigación, se encontraron coeficientes similares a los 
reportados por Rocchi et al. (2017b) y Rodrigues et al. (2019), quienes presentaron coeficientes de consistencia interna alfa de Cronbach entre $.84 \mathrm{y}$ .90 , y .70 y .82 , respectivamente (aunque en este estudio se utilizó el coeficiente de confiabilidad omega de McDonald).

La relevancia principal de esta investigación se centra en la ausencia de instrumentos con evidencias de validez y confiabilidad en el contexto peruano que estudien la relación entrenador(a)-deportista alrededor de las necesidades psicológicas básicas planteadas por la TAD. En las investigaciones dentro del contexto deportivo, el entrenador tiene un rol preponderante ya que es uno de los principales factores que influyen en la calidad de la motivación, el bienestar y el rendimiento de los deportistas (Behzadnia et al., 2018; Hancox et al., 2017; Ntoumanis et al., 2018). Por ello, estudiar esta relación mediante instrumentos adaptados en el contexto nacional, que midan específicamente la interacción entrenador(a)-deportista, resulta ser tan importante. Hasta ahora, diversas investigaciones se han centrado de manera general en los estilos motivacionales de los entrenadores y no en los comportamientos específicos que muestren de acuerdo a cada una de las necesidades psicológicas básicas (Cheon \& Reeve, 2015; Gunnell et al., 2013; Jang et al., 2016; Reeve et al., 2018; Rodríguez, 2017). Por ello, esta adaptación del IBQD en el contexto deportivo peruano podrá permitir el desarrollo de futuras investigaciones en deportistas universitarios.

A pesar de las evidencias de la investigación, hay diversas limitaciones y recomendaciones que tomar en cuenta. Una de las limitaciones es que esta investigación se realizó únicamente con deportistas de universidades de Lima Metropolitana y de disciplinas deportivas diversas. Al respecto, una muestra de estudiantes de diversas universidades del país daría una visión más comprensiva de la relación entre entrenadores(as) y deportistas; no obstante, esta muestra necesitaría tener igual cantidad de participantes para los deportes dado que es posible que haya cambios en la relación entrenador(a)-deportista dependiendo del deporte que se lleva a cabo (e.g. deportes de equipo vs individuales, etc.). Así, se recomienda, para futuras investigaciones, estudiar la equivalencia métrica del IBQD en deportistas universitarios de otras ciudades del Perú que llevan a cabo diversos deportes.

Adicionalmente, resulta importante considerar otros grupos de deportistas que no sean universitarios, como los deportistas profesionales ya que, en este caso, el deporte sería su principal actividad (a diferencia de los deportistas universitarios que, principalmente, son estudiantes que, además, entrenan o hacen deporte). En los deportistas profesionales, la interacción entrenador(a)-deportista y la exigencia de alto rendimiento que implica ser deportista profesional serían diferentes a una relación entrenador(a)-deportista a nivel universitario.

Otra limitación de esta investigación es que se analizó la relación de los comportamientos interpersonales de apoyo y de obstaculización de las NPB con la satisfacción y frustración de las mismas de manera global, por lo que se sugiere estudiar cómo se relacionan los seis tipos de comportamientos interpersonales con la satisfacción y frustración de cada una de las NPB al igual que Rocchi et al. (2017b) y Rodrigues et al. (2019). Así, se podría tener una visión más específica y "fina" de la relación entre entrenadores y deportistas.

Finalmente, este estudio no sólo abre las puertas a investigaciones sobre la interacción deportista-entrenador desde la percepción del deportista, sino que puede estudiarse la percepción del entrenador dado que se podría adaptar al contexto peruano la versión del IBQ de auto-reporte de los entrenadores (IBQ-Self) para analizar la relación entre los comportamientos interpersonales percibidos por los deportistas y los reportados por entrenadores(as), y no solamente reportados por los primeros.

\section{Referencias}

Alarcón, R. (2008). Métodos y diseños de investigación del comportamiento (2a Ed.). Lima: Universidad Ricardo Palma.

American Educational Research Association, American Psychological Association \& National Council on Measurement in Education [AERA, APA \& NCME]. (2014). Standards for Educational and Psychological Testing. Washington, D.C.: AERA. 
Arce, C., de Francisco, C., Andrade, E., Arce, I., \& Raedeke, T. D. (2010). Adaptación española del Athlete Burnout Questionnaire (ABQ) para la medida del burnout en futbolistas. Psicothema, 22(2), 250-255.

Bartholomew, K., Ntoumanis, N., \& Thøgersen-Ntoumani, C. (2010). The controlling interpersonal style in a coaching context: Development and initial validation of a psychometric scale. Journal of Sport and Exercise Psychology, 32, 193-216. https://doi. org/10.1123/jsep.32.2.193

Bartholomew, K., Ntoumanis, N., Ryan, R. M., \& ThøgersenNtoumani, C. (2011). Psychological need thwarting in the sport context: Assessing the darker side of athletic experience. Journal of Sport and Exercise Psychology, 33, 75-102. https://doi.org/10.1123/ jsep.33.1.75

Behzadnia, B., Adachi, P. J. C., Deci, E. L., \& Mohammadzadeh, H. (2018). Associations between students' perceptions of physical education teachers' interpersonal styles and students' wellness, knowledge, performance, and intentions to persist at physical activity: A self-determination theory approach. Psychology of Sport and Exercise, 39, 1019. https://doi.org/10.1016/j.psychsport.2018.07.003

Carpentier, J., \& Mageau, G. A. (2016). Predicting sport experience during training: The role of changeoriented feedback in athletes' motivation, selfconfidence and needs satisfaction fluctuations. Journal of Sport \& Exercise Psychology, 38, 45-58. https://doi.org/10.1123/jsep.2015-0210

Chen, B., Vansteenkiste, M., Beyers, W., Boone, L., Deci, E. L., Van der Kaap-Deeder, J., Duriez, B., Lens, W., Matos, L., Mouratidis, A., Ryan, R. M., Sheldon, K. M., Soenens, B., Van Petegem, S., \& Verstuyf, J. (2015). Basic psychological need satisfaction, need frustration, and need strength across four cultures. Motivation and Emotion, 39, 216-236. https://doi. org/10.1007/s11031-014-9450-1

Cheon, S. H., \& Reeve, J. (2015). A classroom-based intervention to help teachers decrease students' amotivation. Contemporary Educational Psychology, 40, 99-111. https://doi.org/10.1016/j. cedpsych.2014.06.004

De Francisco, C. (2015). Versión reducida del Athlete Burnout Questionnaire (ABQ): propiedades psicométricas preliminares. Revista de Psicología del Deporte, 24(1), 177-183.

Deci, E. L., La Guardia, J. G., Moller, A. C., Scheiner, M. J., \& Ryan, R. M. (2006). On the benefits of giving as well as receiving autonomy support: Mutuality in close friendships. Personality and Social Psychology Bulletin, 32, 313-327. https://doi. org/10.1177/0146167205282148

Deci, E. L., \& Ryan, R. M. (1985). Handbook of selfdetermination research. Rochester, NY: University of Rochester Press.

Delreu, J., Reynders, B., Vande Broek, G., Aelterman, A., De Backer, M., Decroos, S., De Muynck, G. J., Fontaine, J., Fransen, K., van Puyenbroeck, S., Haerens, L., \& Vansteenkiste, M. (2019). Adopting a helicopterperspective towards motivating and demotivating coaching: A circumplex approach. Psychology of Sport \& Exercise, 40, 110-126.

Diener, E., Emmons, R., Larsen, R., \& Griffin, S. (1985). The Satisfaction with Life Scale. Journal of Personality Assessment, 49(1), 71-75. https://doi.org/10.1207/ s15327752jpa4901_13

Federación Deportiva Universitaria del Perú [FEDUP]. (2019). Federación Deportiva Universitaria del Perú. Recuperado de https://www.perufedup.com/ nosotros/

Federación Internacional del Deporte Universitario [FISU]. (2018). Historia FISU. Recuperado de https://www. fisu.net/about-fisu/fisu_history

Forrester, S. (2015). Benefits of Collegiate Recreational Sports Participation: Results from the 2013 NASPA Assessment and Knowledge Consortium Study. Recreational Sports Journal, 39(1), 2-15. https://doi. org/10.1123/rsj.2015-0005

Gargurevich, R. (2010). Propiedades psicométricas de la versión internacional de la Escala de Afecto Positivo y Negativo-forma corta (I-Spanas SF) en estudiantes universitarios. Persona, 13, 31-42.

Garreaud, D. (2019). Estilo de entrenamiento, necesidades psicológicas, vitalidad y oposicionismo desafiante en atletas peruanos (Tesis de Licenciatura). Recuperado de http://hdl.handle. net/20.500.12404/16447

Gunnell, K., Crocker, P. R. E., Wilson, P. M., Mack, D. E., \& Zumbo, B. D. (2013). Psychological need satisfaction and thwarting: A test of basic psychological needs theory in physical activity contexts. Psychology of Sport and Exercise, 14, 599-607. https://doi. org/10.1016/j.psychsport.2013.03.007

Haerens, L., Aelterman, N., Vansteenkiste, M., Soenens, B., \& Van Petegem, S. (2015). Do perceived autonomysupportive and controlling teaching relate to physical education students' motivational 
experiences through unique pathways? Distinguishing between the bright and dark side of motivation. Psychology of sport and Exercise, 16(3), 26-36. https://doi.org/10.1016/j. psychsport.2014.08.013

Hagger, M., \& Chatzisarantis, N. (2007). Intrinsic motivation and self-determination in exercise and sport. Leeds, UK: Human Kinetics Europe Ltd.

Haines, D. J. (2001). Undergraduate Student Benefits from University Recreation. Recreation Sports Journal, 25(1), 25-33. https://doi.org/10.1123/nirsa.25.1.25

Hambleton, R. K. (1994). Guidelines for adapting educational and psychological tests: A progress report. European Journal of Psychological Assessment, 10(3), 229-244

Hambleton, R. K., \& Zenisky, A. L. (2011). Translating and adapting tests for cross-cultural assessments. En D. Matsumoto \& F. J. R. van de Vijver (Eds.), Crosscultural research methods in psychology (pp. 4670). New York: Cambridge University Press.

Hancox, J. E., Quested, E., Ntoumanis, N., \& ThøgersenNtoumani, C. (2017). Putting self-determination theory into practice: application of adaptive motivational principles in the exercise domain. Qualitative Research in Sport, Exercise and Health, 10(1), 75-91. https://doi. org/10.1080/2159676X.2017.1354059

Hein, V., Koka, A., \& Hagger, M. S. (2015). Relationships between perceived teachers' controlling behaviour, psychological need thwarting, anger and bullying behaviour in high-school students. Journal of Adolescence, 42, 103-114. https://doi.org/10.1016/j. adolescence.2015.04.003

Hu, L. T., \& Bentler, P. M. (1999). Cutoff Criteria for Fit Indexes in Covariance Structure Analysis: Conventional Criteria versus New Alternatives. Structural Equation Modeling, 6(1), 1-55. https://doi. org/10.1080/10705519909540118

IBM Corp. (2017). IBM SPSS Statistics for Windows, Version 25.0 [Software de computador]. Armonk, NY: IBM Corp.

Jang, H., Kim, E. J., \& Reeve, J. (2016). Why students become more engaged or more disengaged during the semester: A self-determination theory dual-process model. Learning and Instruction, 43, 27-38. https:// doi.org/10.1016/j.learninstruc.2016.01.002

JASP Team. (2020). JASP Version 0.13.1 [Software de computador]. Amsterdam-Centrum, AMS: Universiteit van Amsterdam.

Jöreskog, K. G., \& Sörbom, D. (2005). LISREL 8.72: Interactive
LISREL for MS Windows [Software de computador]. Lincolnwood, IL: Scientific Software International, Inc.

Jöreskog, K. G., \& Sörbom, D. (1996). PRELIS 2 user's reference guide: A program for multivariate data screening and data summarization: A preprocessor for LISREL. Chicago, IL: Scientific Software International.

Katz, M. H. (2006). Multivariable analysis (2nd Ed.). Cambridge, CB: Cambridge University Press.

Kline, R. B. (2016). Principles and Practice of Structural Equation Modeling (Fourth Ed.). New York, NY: The Guilford Press.

López-Walle, J. M., Balaguer, I., Castillo, I., \& Tristán, J. L. (2012). Autonomy support, Basic psychological needs and Well-being in Mexican Athletes. The Spanish Journal of Psychology, 15(3), 1283-1292. https://doi.org/10.5209/rev_SJOP.2012.v15.n3.39414

Lower, L. M., Turner, B. A., \& Petersen, J. C. (2013). A Comparative Analysis of Perceived Benefits of Participations between Recreational Sport Programs. Recreational Sports Journal, 37(1), 66-83. https://doi.org/10.1123/rsj.37.1.66

Mageau, G., Ranger, F., Joussemet, M., Koestner, R., Moreau, E., \& Forest, J. (2015). Validation of the Perceived Parental Autonomy Support Scale (P-PASS). Canadian Journal of Behavioural Science, 47, 251262. https://doi.org/10.1037/a0039325

Marsh, H. W., Hau, K., \& Wen, Z. (2004). In search of golden rules: Comment on hypothesis testing approaches to setting cutoff values for fit indexes and dangers in overgeneralizing $\mathrm{Hu}$ and Bentler's (1999) findings. Structural Equation Modeling: $A$ Multidisciplinary Journal, 11(3), 320-341. https:// doi.org/10.1207/s15328007sem1103_2

Martínez, P. (2004). Perspectiva temporal futura y satisfacción con la vida a lo largo del ciclo vital. Revista de Psicología de la PUCP, 22(2), 217-252. https://doi.org/10.18800/psico.200402.003

Matos, L., \& Gargurevich, R. (2020). La escala de necesidades psicológicas básicas versión corta. Manuscrito en preparación.

Maydeu-Olivares, A. (2017). Maximum Likelihood Estimation of Structural Equation Models for Continuous Data: Standard Errors and Goodness of Fit. Structural Equation Modeling: A Multidisciplinary Journal, 24(3), 2-12. https://doi.org/10.1080/10705511.2016.1269606

McDonald, R. P. (1999). Test theory: A unified treatment. Mahwah, NJ: Lawrence Erlbaum Associates Inc. 
Muñiz, J., Elosua, P., \& Hambleton, R. K. (2013). Directrices para la traducción y adaptación de los tests: segunda edición. Psicothema, 25(2), 151-157. https:// doi.org/10.7334/psicothema2013.24

Norman, G. (2010). Likert scales, levels of measurement and the "laws" of statistics. Advances in Health Sciences Education, 15, 625-632. https://doi.org/10.1007/ s10459-010-9222-y

Ntoumanis, N., Guerrero, M. D., Gadeke, C., \& ThøgersenNtoumani, C. (2018). Do exerciser weight status and perceived motivation predict instructors' motivation and beliefs about the exerciser? A test of motivation contagion effects. Body Image, 26, 10-18. https://doi.org/10.1016/j.bodyim.2018.05.001

Raedeke, T. D., \& Smith, A. L. (2001). Development and preliminary validation of an athlete burnout measure. Journal of Sport \& Exercise Psychology, 23, 281-306. https://doi.org/10.1123/jsep.23.4.281

Raykov, T. (1997). Scale reliability, Cronbach's coefficient alpha, and violations of essential tau- equivalence with fixed congeneric components. Multivariate behavioral research, 32(4), 329-353.

Reeve, J. (2009). Why teachers adopt a controlling motivating style toward students and how they can become more autonomy supportive. Educational Psychologist, 44(3), 159-175. https:// doi.org/10.1080/00461520903028990

Reeve, J., Jang, H. R., \& Jang, H. (2018). Personality-based antecedents of teachers' autonomy-supportive and controlling motivating styles. Learning and Individual Differences, 62, 12-22. https://doi. org/10.1016/j.lindif.2018.01.001

Reinboth, M., Duda, J. L., \& Ntoumanis, N. (2004). Dimensions of coaching behavior, need satisfaction, and the psychological and physical welfare of young athletes. Motivation and Emotion, 28, 297-313. https://doi.org/10.1023/ B:MOEM.0000040156.81924.b8

Rocchi, M., Pelletier, L., Cheung, S., Baxter, D., \& Beaudry, S. (2017a). Assessing need-supportive and need-thwarting interpersonal behaviours: The Interpersonal Behaviours Questionnaire (IBQ). Personality and Individual Differences, 104, 423433. https://doi.org/10.1016/j.paid.2016.08.034

Rocchi, M., Pelletier, L., \& Desmarais, P. (2017b). The validity of the Interpersonal Behaviors Questionnaire (IBQ) in sport. Measurement in Physical Education and Exercise Science, 21(1), 15-25. https://doi. org/10.1080/1091367X.2016.1242488
Rodrigues, F., Pelletier, L., Neiva, H. P., Teixeira, D. S., Cid, L., \& Monteiro, D. (2019). Initial validation of the Portuguese version of the Interpersonal Behavior Questionnaire (IBQ \& IBQ-Self) in the context of exercise: Measurement invariance and latent mean differences. Current Psychology, 1-12. https://doi. org/10.1007/s12144-019-00374-y

Rodríguez, J. M. (2017). Estilo motivacional, necesidades psicológicas básicas, tipos de motivación, vitalidad y agotamiento en deportistas escolares (Tesis de Licenciatura). Recuperado de http://hdl.handle. net/20.500.12404/11844

Ryan, R. M., \& Deci, E. L. (2000). Self-determination theory and the facilitation of intrinsic motivation, social development, and well-being. American Psychologist, 55(1), 68-78. http://doi. org/10.1037110003-066X.55.1.68

Ryan, R. M., \& Deci, E. L. (2017). Self-determination theory: Basic psychological needs in motivation, development, and wellness. Guilford Publications.

Ryan, R. M., \& Frederick, C. (1997). On energy, personality, and health: Subjective vitality as a dynamic reflection of well-being. Journal of Personality, 65(3), 529-565. https://doi.org/10.1111/j.1467-6494.1997.tb00326.x

Ryan, R. M., Patrick, H., Deci, E. L., \& Williams, G. C. (2008). Facilitating health behavior change and its maintenance: Interventions based on selfdetermination theory. The European Health Psychologist, 10(1), 2-5.

Sevil, J., Abós, A., Sanz, M., \& García-González, L. (2018). El "Lado Claro" y El "Lado Oscuro" de la Motivación en Educación Física: Efectos de una Intervención en una Unidad Didáctica de Atletismo. Revista Iberoamericana de Diagnóstico y Evaluación - e Avaliação Psicológica, 46(1), 93-107. https://doi. org/10.21865/RIDEP46.1.07

Simms, L. J., Watson, D., \& Doebbeling, B. N. (2002). Confirmatory Factor Analyses of Posttraumatic Stress Symptoms in Deployed and Nondeployed Veterans of the Gulf War. Journal of Abnormal Psychology, 111(4), 637-647. https://doi. org/10.1037/0021-843X.111.4.637

Steiger, J. H. (2007). Understanding the limitations of global fit assessment in structural equation modeling. Personality and Individual Differences, 42(5), 89398. https://doi.org/10.1016/j.paid.2006.09.017

Tabachnick, B. G., \& Fidell, L. S. (1996). Using Multivariate Statistics (3rd ed.). New York: Harper Collins.

Thompson, E. R. (2007). Development and validation of an 
internationally reliable short-form of the Positive and Negative Affect Schedule (PANAS). Journal of Cross-Cultural Psychology, 38, 227-242. https://doi. org/10.1177/0022022106297301

Tomlinson, A. (2010). A Dictionary of Sports Studies. NY: Oxford University Press Inc.

Vallerand, R. (2001). A hierarchical model of intrinsic and extrinsic motivation in sport and exercise. En G. C. Roberts (Ed.), Advances in motivation in sport and exercise (pp. 263-319). Champaign, IL: Human Kinetics.

Vansteenkiste, M., \& Ryan, R. M. (2013). On psychological growth and vulnerability: Basic psychological need satisfaction and need frustration as a unifying principle. Journal of Psychotherapy Integration, 23(3), 263-280. https://doi.org/10.1037/a0032359

Weinberg, R., \& Gould, D. (2011). Foundations of Sport and Exercise Psychology (6th Ed.). Champaign, IL: Human Kinects.

West, S. G., Finch, J. F., \& Curran, P. J.(1995). Structural equation models with nonnormal variables: Problems and remedies. En R. H. Hoyle (Ed.), Structural equation modeling: Concepts, issues and applications (pp. 5675). Newbery Park, CA: Sage.

RIDU / Revista Digital de Investigación en Docencia Universitaria / e-ISSN: 2223-2516

(c) Los autores. Este artículo es publicado por la Revista Digital de Investigación en Docencia Universitaria del Área de Institutional Research and Effectiveness de la Dirección de Aseguramiento de la Calidad, Universidad Peruana de Ciencias Aplicadas. Este es un artículo de acceso abierto, distribuido bajo los términos de la LicenciaCreativeCommons Atribución-CompartirIgual 4.0 Internacional.( http://creativecommons.org/licenses/by-sa/4.o/), que permite el uso no comercial, distribución y reproducción en cualquier medio, siempre que la obra original sea debidamente citada. "Este es un artículo de acceso abierto, distribuido bajo los términos de la LicenciaCreativeCommons Atribución 4.0 Internacional (CC BY 4.0) (https://creativecommons.org/licenses/by/4.0/ deed.es), que permite el uso, distribución y reproducción en cualquier medio, siempre que la obra original sea debidamente citada." 